\title{
El tratamiento farmacológico en pacientes hipertensos leves no demostró eficacia en reducir morbimortalidad
}

\author{
Drug therapy in mild hypertensive patients did not demonstrate efficacy in reducing morbidity
} and mortality

Diao D y Col. Cochrane Database of Systematic Reviews 2012, Issue 8. Art No.: CD006742.

\section{Objetivos}

Cuantificar el efecto del tratamiento con drogas antihipertensivas sobre la morbimortalidad y efectos adversos en pacientes sanos con hipertensión arterial (HTA) leve.

\section{Fuente y selección de datos}

DARE, Cochrane Central (hasta final de 2011), Medline (1948 a Mayo 2011) y Embase (1980 a Mayo 2011); referencias de los textos encontrados y contacto con autores. La búsqueda se limitó a incluir ensayos clínicos controlados y aleatorizados $(E C C A)^{*}$ de mínimo un año de duración con las siguientes características: inclusión de al menos $80 \%$ de participantes (hombres y mujeres mayores de 18 años) con HTA en estadio uno o estudios que proveyeran datos de pacientes individuales; HTA leve definida como presión arterial sistólica de 140 a 159 mmHg o presión arterial diastólica de 90 a $99 \mathrm{mmHg}$; participantes sin evidencia de enfermedad cardiovascular al inicio del estudio; grupo intervención tratado con drogas antihipertensivas como monoterapia o en adición a otra droga en abordaje escalonado, y grupo control asignado a placebo o no tratamiento.

Se agruparon todos los datos y se calculó el riesgo relativo* (RR) para cada resultado de interés.

\section{Resultados Principales}

Se analizaron cuatro estudios de regular calidad científica. Los principales resultados se resumen en la tabla 1. En el mejor escenario posible, una reducción del riesgo absoluto* de $0,25 \%$ para mortalidad y de $0,78 \%$ para eventos cardiovasculares totales a cinco años tendría un número necesario a tratar de 400 y 128 pacientes para prevenir una muerte o un evento cardiovascular respectivamente. Por otro lado un $9 \%$ de los pacientes sufrirían efectos adversos por los cuales abandonarían la terapia.

Tabla 1: efecto del tratamiento antihipertensivo en pacientes con hipertensión arterial leve.

\begin{tabular}{l|c|c|c|} 
& N $^{0}$ de estudios & N & RR (IC95\%) \\
\hline Mortalidad Global & 4 & 8.912 & $0,85(0,63$ a 1,15) \\
\hline Total de eventos cardiovasculares & 3 & 7.080 & $0,51(0,24$ a 1,08$)$ \\
\hline Enfermedad Coronaria & 3 & 7.080 & $1,12(0,80$ a 1,57$)$ \\
\hline Accidente cerebrovascular & 3 & 7.080 & $0,97(0,72$ a 1,32$)$ \\
\hline Abandonos por efectos adversos & 1 & 17.354 & $4,80(4,14$ a 5,57$)$ \\
\hline
\end{tabular}

\section{Conclusiones}

El tratamiento farmacológico antihipertensivo de pacientes adultos con HTA leve no demostró reducir la morbimortalidad en los ECCA. El tratamiento fue discontinuado en un $9 \%$ de los participantes a causa de efectos adversos. Se requerirán más ECCA en este prevalente grupo para conocer si los beneficios del tratamiento superan los daños.

Fuente de financiamiento/ conflicto de interés de los autores: no referido.

\section{Comentario}

Resulta sorprendente, o al menos inesperado, encontrar estos resultados para una práctica clínica tan difundida e instaurada casi sin cuestionamientos, en esta "era" que busca logar el "control" de todos los factores de riesgo cardiovascular.

Teniendo en cuenta las recomendaciones de guías de práctica clínica que marcaron la senda en los últimos años, como el reporte JNC VII ${ }^{1}$, uno podría pensar que es ridículo y hasta poco ético no tratar la HTA (incluido el actualmente denominado estadio I). Sin embargo en línea con las conclusiones de la presente revisión, la reciente y última guía NICE sobre evaluación y manejo de la $\mathrm{HTA}^{2}$ propone ofrecer tratamiento farmacológico a las personas menores de 80 años con HTA estadio I que tengan evidencia de daño de órgano blanco (como enfermedad cardiovascular establecida, enfermedad renal, diabetes) o un riesgo cardiovascular a diez años que supere el $20 \%$.

Respecto de los resultados de la presente revisión debemos hacer algunas salvedades: la evidencia encontrada es de moderada calidad; y los cuatro estudios incluidos fueron realizados entre las décadas de los 70 y 80 , utilizando drogas y dosis que ya no se utilizan en la actualidad (clorotiazida, bendroflutiazida, propranolol, clortalidona, metildopa, pindolol, atenolol, reserpina). Así, los efectos adversos podrían haber sido mayores a los que ocurren con las drogas usadas en la actualidad. Por otro lado, los eventos que se miden son de baja incidencia en esa población por lo cual el poder* para demostrar beneficios debería ser mucho mayor. Según lo observado por la guía NICE previamente mencionada ${ }^{2}$ los hipertensos incluidos en esos estudios podrían no serlo en realidad.

\section{Conclusiones del comentador}

Luego de analizar esta revisión podemos afirmar que la evidencia no es concluyente en cuanto a reducción de morbimortalidad con un porcentaje significativo de abandono de tratamiento debido a efectos adversos.

Sería ético realizar en la actualidad un ensayo clínico que trate de demostrar estos beneficios. Aun de ser reales, los resultados de este estudio no modificarían el hecho de recomendar medidas no farmacológicas donde se fomenten cambios en el estilo de vida.

Agustin Segalini [ Servicio de Medicina Familiar y Comunitaria del Hospital Italiano de Buenos Aires. agustin.segalini@hospitalitaliano.org.ar ]

Segalini A. El tratamiento farmacológico en pacientes hipertensos leves no demostró eficacia en reducir morbimortalidad. Evid Act Pract Ambul OctDic 2012;15(4):127. Comentado de: Diao D, Wright JM, Cundiff DK, et al. Pharmacotherapy for mild hypertension. Cochrane Database of Systematic Reviews 2012, Issue 8. Art No.: CD006742. PMID: 22895954.

\section{Referencias}

1. Chobanian AV, Bakris GL, Black HR, et al. The Seventh Report of the Joint National Committee on Prevention, Detection, Evaluation, and Treatment of High Blood Pressure: the JNC 7 report. JAMA 2003; 289:2560.

2. Grande Ratti Maria Florencia, Augustovski, Federico. Manejo de la hipertensión del adulto en Atención Primaria. Evid Act Pract Ambul Ene-Mar 2012;15(1):34-36. Comentado de: National Institute for Health and Clinical Excellence. Hypertension: clinical management of primary hypertension in adults (update). Clinical guideline 127, August 2011. Disponible en URL: http://guidance.nice.org.uk/CG127 